\title{
Electronic and structural properties of graphene-based transparent and conductive thin film electrodes
}

\author{
A. Vollmer $\cdot$ X.L. Feng $\cdot$ X. Wang $\cdot$ L.J. Zhi $\cdot$ K. Müllen \\ N. Koch · J.P. Rabe
}

Received: 27 August 2008 / Accepted: 6 October 2008 / Published online: 14 October 2008

(C) Springer-Verlag 2008

\begin{abstract}
We demonstrate that graphene-based transparent and conductive thin films (GTCFs), fabricated by thermal reduction of graphite oxide, have very similar electronic and structural properties as highly oriented pyrolytic graphite (HOPG). Electron spectroscopy results suggest that the GTCFs are also semi-metallic and that the individual graphene sheets of the film are predominantly oriented parallel to the substrate plane. These films may therefore be considered as a technologically relevant analogue to HOPG electrodes, which cannot be easily processed into thin films.
\end{abstract}

PACS 61.05.Cj · 73.61.-r $\cdot 79.60 . \mathrm{Bm}$

\section{Introduction}

Recently, graphene-based thin films have attracted considerable attention as they are chemically robust, exhibit high electrical conductivity and good optical transparency in the visible and near-infrared region [1-6]. This renders these films interesting as electrodes for various applications, e.g., in organic electronic devices $[5,6]$ or for spectroelectrochemical studies. Other transparent and conductive thin film

\section{A. Vollmer}

Berliner Elektronenspeicherring-Gesellschaft für

Synchrotronstrahlung m.b.H., 12489 Berlin, Germany

X.L. Feng $\cdot$ X. Wang $\cdot$ L.J. Zhi $\cdot$ K. Müllen

Max Planck Institute for Polymer Research, Ackermannweg 10,

55128 Mainz, Germany

N. Koch $(\bowtie) \cdot$ J.P. Rabe

Institut f. Physik, Humboldt-Universität zu Berlin, Newtonstr. 15, 12489 Berlin, Germany

e-mail: norbert.koch@ physik.hu-berlin.de materials, such as indium tin oxide or fluorine tin oxide, however, are problematic for several applications [5, 7]. The limited availability of the element indium on earth, the poor transparency of the oxides in the near-infrared, and their instability in the presence of acid or base motivate the search for alternatives. Graphene films fabricated from exfoliated graphite oxide, followed by thermal reduction, appear as interesting candidates for electrode applications because of their high conductivity (up to $550 \mathrm{~S} / \mathrm{cm}$ have been demonstrated) and transmission of $70 \%$ in the wavelength range of 1000-3000 nm [5]. The existence of graphene sheets in such thin films has been evidenced by high-resolution transmission electron microscopy, and bulk Raman spectra revealed the similarity of reduced graphene-oxide with carbon nanotubes or graphite with defects [5, 8]. It can thus be expected that such graphene films have electronic properties similar to various other $\mathrm{sp}^{2}$ hybridized carbon-based materials, such as nanotubes or graphite. However, rather little is known about the surface electronic properties of such graphenebased films and the surface orientation of graphene sheets over extended areas.

In this report we show that the surface electronic and structural properties of graphene-based films fabricated by thermal reduction of exfoliated graphite oxide are very similar to those of highly oriented pyrolytic graphite (HOPG). Using near-edge X-ray absorption fine structure (NEXAFS) spectroscopy and angle-resolved ultraviolet photoelectron spectroscopy (ARUPS), the overall shape of the obtained spectra allowed identifying chemical species, and the analysis of incidence-angle dependent peak intensities, attributed to particular transitions in NEXAFS and ARUPS, provided information on the geometry. X-ray photoelectron spectroscopy (XPS) investigations corroborated these findings. 


\section{Experimental}

Photoemission and absorption experiments were performed at the end-station SurICat (beamline PM4) at the synchrotron light source BESSY (Berlin, Germany) [9]. The ultrahigh vacuum (UHV) system consists of interconnected sample preparation/load lock (base pressure: $1 \times 10^{-8} \mathrm{mbar}$ ) and analysis (base pressure: $1 \times 10^{-10} \mathrm{mbar}$ ) chambers. Excitation energy was $45 \mathrm{eV}$ for UPS and $630 \mathrm{eV}$ for XPS; the spectra were collected with a hemispherical electron energy analyzer (Scienta SES 100) with $100 \mathrm{meV}$ (UPS) and $250 \mathrm{meV}$ (XPS) energy resolutions. X-ray absorption of the carbon K-edge was collected in the energy range between $275 \mathrm{eV}$ and $325 \mathrm{eV}$ in steps of $100 \mathrm{meV}$ (energy resolution $250 \mathrm{meV}$ ) at three different angles of incidence $\alpha=0^{\circ}, 45^{\circ}$, and $70^{\circ}$, respectively, where $\alpha=0^{\circ}$ corresponds to the electric field vector of the incident light being perpendicular to the surface normal. The NEXAFS signal was obtained via the sample current and normalized to the beamline photon flux transmission by forming the ratio of the absorption of the sample and previously recorded spectra obtained from a freshly cleaned gold single crystal. HOPG (GE Advanced Ceramics, USA) was cleaved in air immediately before loading into the UHV system. Graphene-based transparent and conductive films (GTCFs) were fabricated by dip coating a quartz substrate with a graphite oxide solution, followed by heat treatment at $1100^{\circ} \mathrm{C}[5]$.

\section{Results and Discussion}

In XPS only the $\mathrm{C} 1 \mathrm{~s}$ signal was clearly observed for HOPG in a wide range scan of binding energies up to $580 \mathrm{eV}$ (see Fig. 1). Traces of oxygen could be seen in a close-up of the $O$ $1 \mathrm{~s}$ region, attributed to contamination of $\mathrm{HOPG}$ by $\mathrm{O}_{2}$ and water due to the exposure to air prior to loading the sample into the UHV system. For the GTCF sample, however, strong signals from $\mathrm{O} 1 \mathrm{~s}$ and $\mathrm{Si} 2 \mathrm{p}$ core levels in addition

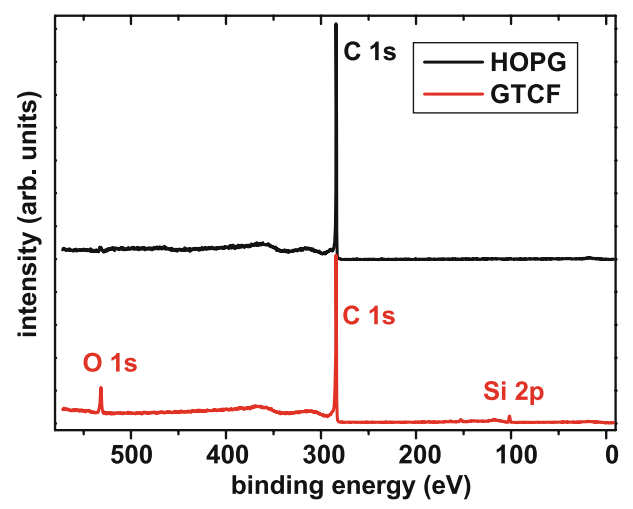

Fig. 1 XPS survey spectra of HOPG (top) and GTCF (bottom). Photon energy was $630 \mathrm{eV}$ to the predominant $\mathrm{C} 1 \mathrm{~s}$ could be detected. The $\mathrm{Si} 2 \mathrm{p}$ and most of the $\mathrm{O} 1 \mathrm{~s}$ signal is attributed to the quartz substrate. The average graphene film thickness was $22 \mathrm{~nm}$ (as determined from atomic force microscopy measurements upon scraping trenches into the film) and should thus completely attenuate the XPS signal from the substrate. We conclude that the films exhibited either small pinholes or had localized scratches due to sample handling, which led to the $\mathrm{Si}$ and $\mathrm{O}$ signal in Fig. 1. The presence of only one narrow C 1s peak for the GTCF sample indicates chemical homogeneity throughout the film. XPS therefore indicates a high chemical resemblance of HOPG and GTCF.

A comparison of the valence band spectra of HOPG and GTCF reveals a pronounced similarity of the two specimens, as can be seen in Fig. 2(a). However, there are some differences in peak intensity distribution. Most notably, the peaks near $13 \mathrm{eV}$ and $16 \mathrm{eV}$ binding energy observed in the GTCF spectra could be assigned to the $\mathrm{C} 2 \mathrm{~s}$ valence emission from $\mathrm{sp}^{3}$ hybridized carbon species [10]. The presence of such types of defects in the otherwise $\mathrm{sp}^{2}$ hybridized carbon in graphite has been proposed to exist in thermally reduced graphite oxide samples on the edges of zigzag edges [8].
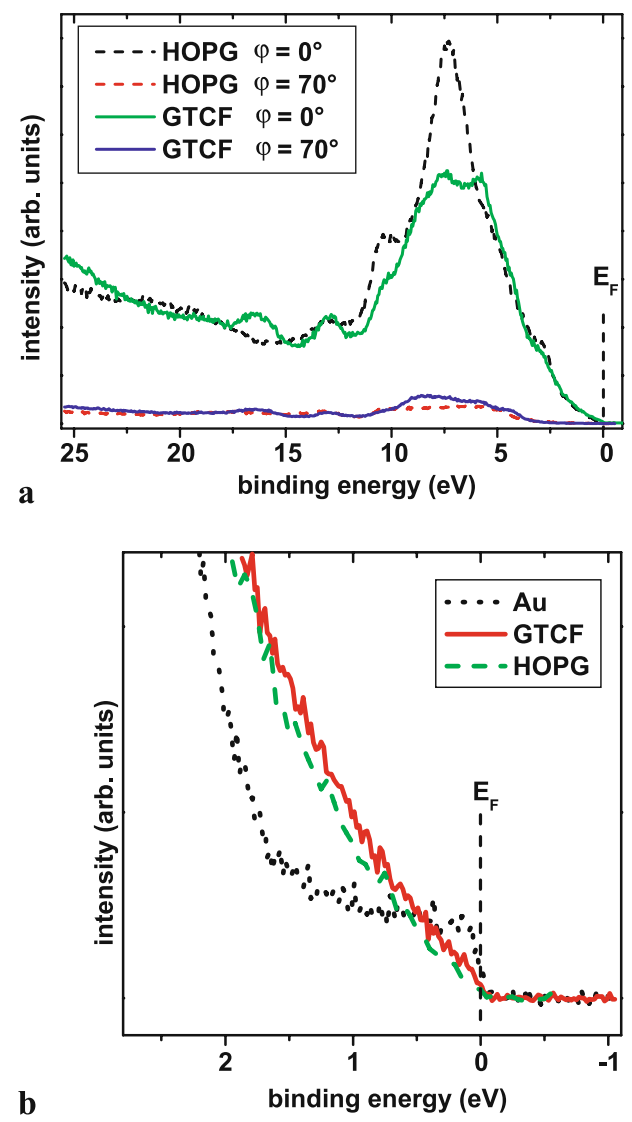

Fig. 2 UPS spectra of HOPG and GTCF. (a) Valence band for take-off angles $\varphi$ as indicated, (b) near- $E_{\mathrm{F}}$ region for $\varphi=0^{\circ}$; for comparison, the Fermi-edge of $\mathrm{Au}$ is shown. The spectra are plotted relative to the Fermi level, the photon energy was $45 \mathrm{eV}$ 
Most importantly, the GTCF exhibits the same density of occupied states close to the Fermi level $\left(E_{\mathrm{F}}\right)$ as HOPG. For both samples, the density of valence states extends to $E_{\mathrm{F}}[$ Fig. 2(b)] at room temperature, indicative of the semimetallic nature of both materials. The Fermi edge of clean gold is also shown for comparison. Since HOPG exhibits a strong angular intensity dependence in UPS due to its high orientation of the $\pi$-orbitals (parallel to the surface normal), the dependence of photoemission intensity on the take-off angle $\varphi$ was investigated for both HOPG and GTCF [see Fig 2(a)]; $\varphi=0^{\circ}$ indicates electron emission normal to the sample surface. The dependence was as expected for graphite and equally found for the GTCF, indicating a very similar graphene sheet orientation and also pointing out that surface contaminations (e.g., by disordered saturated hydrocarbons, water, etc.) are minimal for both samples.

To further evidence that the reduced graphite-oxide film has graphitic character, angle dependent NEXAFS experiments were carried out (Fig 3). The spectral features originate from transitions of $\mathrm{C} 1 \mathrm{~s}$ electrons into states of $\pi$ - and $\sigma$-symmetry, and dipole selection rules can be used to understand the angular dependence of the peak intensities (for HOPG: $\mathrm{C} 1 \mathrm{~s} \rightarrow \pi^{*}$ intense for incident electric field vector parallel to the surface normal; $\mathrm{C} 1 \mathrm{~s} \rightarrow \sigma^{*}$ intense for the vector perpendicular to the surface normal) [11, 12]. For
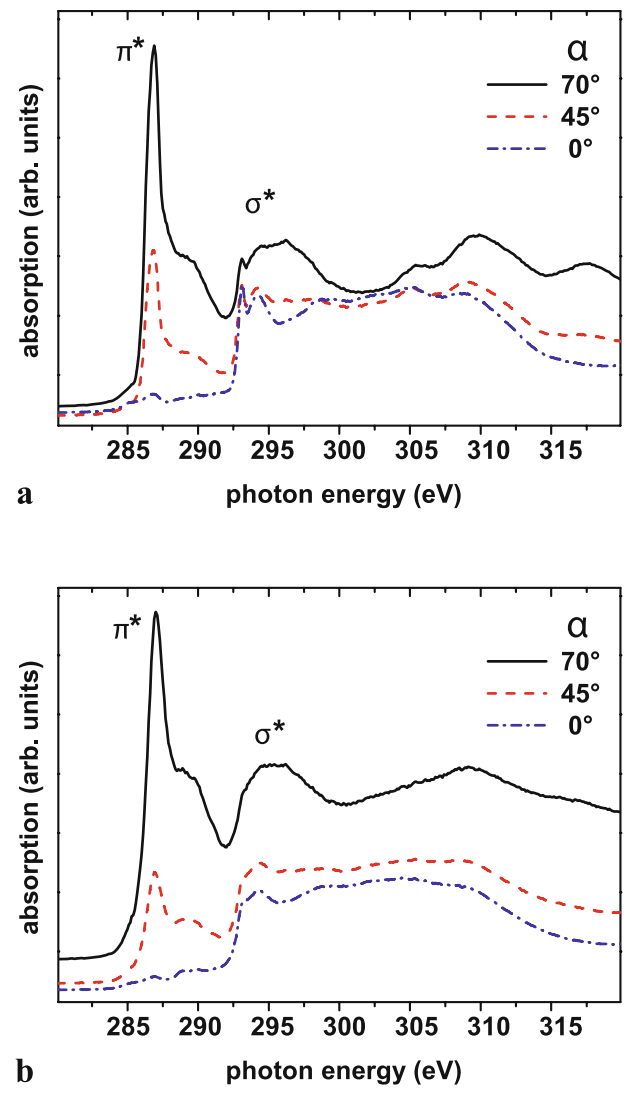

Fig. 3 Angle-dependent NEXAFS spectra of (a) HOPG and (b) GTCF the electric field, the vector of the incident light perpendicular to the $\pi^{*}$ orbitals of graphite the absorption is minimal; in our experimental set-up this corresponds to $\alpha=0^{\circ}$. For $\alpha=70^{\circ}$, the electric field vector is almost parallel to the $\pi^{*}$ orbitals, and the absorption is significantly stronger. NEXAFS spectra of HOPG [Fig. 3(a)] show the well-known signatures of absorption into $\pi^{*}$ states at $285 \mathrm{eV}$ and $\sigma^{*}$ states at $292 \mathrm{eV}$, respectively, and the expected angular dependence as outlined above. Likewise, the absorption spectra of GTCF [Fig. 3(b)] exhibit the same spectral features as observed for HOPG, and importantly also the same angular dependence of the $\pi^{*}$ and $\sigma^{*}$ absorptions. This is a direct evidence that (i) $\mathrm{C}$ in GTCF is in the same configuration as in graphite, and (ii) the individual graphene sheets are predominately oriented parallel to the sample surface, as in HOPG. The results obtained from NEXAFS therefore corroborate and verify the conclusion drawn from the ARUPS and XPS investigations.

\section{Conclusion}

Summarizing the XPS, UPS, and NEXAFS results, it has been shown that graphene-based thin films fabricated by thermal reduction of graphite oxide have very similar electronic and structural properties as HOPG. However, the presence of some $\mathrm{sp}^{3}$ hybridized carbon species is indicated by surface sensitive UPS. The transparent and highly conductive graphene film is likely semi-metallic, and the individual graphene sheets of the film are predominantly oriented parallel to the substrate plane. It is therefore possible to use such graphene-based films as technologically relevant analogue to HOPG, which cannot be easily processed into thin films.

Acknowledgements We thank Constants Weber for AFM measurements and Dörthe M. Eisele for discussions. This work was in part supported by the DFG (SFB 658 "Elementary processes in molecular switches at surfaces"), the EC (project "ICONTROL"; ECSTREP-033197) and the Max Planck Society through the program ENERCHEM. N.K. acknowledges support by the Emmy Noether program (DFG).

\section{References}

1. A.K. Geim, K.S. Novoselov, Nat. Mater. 6, 183 (2007)

2. K.S. Novoselov, A.K. Geim, S.V. Morozov, D. Jiang, Y. Zhang, S.V. Dubonos, I.V. Grigorieva, A.A. Firsov, Science 306, 666 (2004)

3. C. Gomez-Navarro, R.T. Weitz, A.M. Bittner, M. Scolari, A. Mews, M. Burghard, K. Kern, Nano Lett. 7, 3499 (2007)

4. S. Stankovich, D.A. Dikin, G.H.B. Dommett K, M. Kohlhaas, E.J. Zimney, E.A. Stach, R.D. Piner, S.T. Nguyen, R.S. Ruoff, Nature 442, 282 (2006)

5. X. Wang, L.J. Zhi, K. Müllen, Nano Lett. 8, 323 (2008) 
6. S.R.C. Vivekchand, C.S. Rout, K.S. Subrahmanyam, K.S. Subrahmanyam, A. Govindaraj, C.N.R. Rao, J. Chem. Sci. 120, 9 (2008)

7. S. Donner, H.W. Li, E.S. Yeung, M.D. Porter, Anal. Chem. 78, 2816 (2006)

8. K.N. Kudin, B. Ozbas, H.C. Schniepp, R.K. Prud'homme, I.A. Aksay, R. Car, Nano Lett. 8, 36 (2008)

9. A. Vollmer, O.D. Jurchescu, I. Arfaoui, I. Salzmann, T.T.M. Palstra, P. Rudolf, J. Niemax, J. Pflaum, J.P. Rabe, N. Koch, Eur. Phys. J. E 17, 339 (2005)
10. R. Weber, B. Winter, I.V. Hertel, B. Stiller, S. Schrader, L. Brehmer, N. Koch, J. Phys. Chem. B 107, 7768 (2003)

11. C. Jäger, Th. Henning, R. Schlögl, O. Spillecke, J. Non-Cryst. Solids 258, 161 (1999)

12. R.A. Rosenberg, P.J. Love, V. Rehn, Phys. Rev. B 33, 4034 (1986) 